\title{
Time and length scales for diffusion in liquids
}

\author{
A. M. Berezhkovskii ${ }^{1, *}$ and G. Sutmann ${ }^{2, \dagger}$ \\ ${ }^{1}$ Center for Information Technology, National Institutes of Health, Bethesda, Maryland 20892 \\ ${ }^{2}$ Central Institute for Applied Mathematics and John von Neumann Institute for Computing, Research Centre Jülich, \\ D-52425 Jülich, Germany
}

(Received 24 August 2001; revised manuscript received 28 March 2002; published 14 June 2002)

\begin{abstract}
The first six even moments of the displacement of a molecule in water and an atom in liquid argon are found by molecular dynamics simulations and compared with the moments predicted by diffusion theory. We find a noticeable difference between the moments higher than the second. The ratio between predicted and calculated moments approaches unity as $1 / t$ for times larger than $10 \mathrm{ps}$. Continuous time random walk is used to explain this slow approach of the moments to their diffusion limit.
\end{abstract}

DOI: 10.1103/PhysRevE.65.060201

PACS number(s): 66.10.Cb, 02.70.Ns, 05.40.Fb, 05.60.Cd

Motion of individual molecules in liquids is a classic example of the many-body problem. It is well known, however, that at sufficiently large times this motion is well described in terms of ordinary diffusion. Thus, at these times the manybody problem reduces to a one-body stochastic problem in which all many-body effects are hidden into a single number, the diffusion constant. The question we analyze in this paper is which times can be considered as sufficiently large to comfortably use the language of diffusion? This question may be of importance for understanding the processes occuring on picosecond time scales and angstrom length scales.

It should be noted that in contrast to gases and crystalline solids there is no well established picture of molecular motion that underlines self-diffusion in liquids [1-4]. Our analysis suggests that at intermediate times the molecular motion can be described in terms of a random walk, which is due to jumps of the liquid's configuration from one local minimum of the multidimensional potential energy surface to another. The idea that diffusion in liquids occurs as a result of such jumps was put forward by Zwanzig [5]. Rabani, Gezelter, and Berne recently used the Zwanzig model to calculate the self-diffusion constant of argon and $\mathrm{CS}_{2}$ in regular and supercooled regimes [6]. They found excellent agreement between the self-diffusion constants calculated on the basis of the Zwanzig hopping model and those calculated via the Einstein relation, $D=\lim _{t \rightarrow \infty}\left\langle[\mathbf{r}(t)-\mathbf{r}(0)]^{2}\right\rangle / 6 t$, over a wide range of temperatures and densitites.

Our analysis is based on particle trajectories calculated by means of molecular dynamics (MD) simulations for two different model systems: a Lennard-Jones liquid (argon) and $\mathrm{SPC} / \mathrm{E}$ water [7]. In the case of argon we have simulated 2048 atoms at a temperature $T=94 \mathrm{~K}$ and a density $\rho$ $=1.36 \mathrm{~g} / \mathrm{cm}^{3}$. In the case of water we considered $256 \mathrm{~mol}-$ ecules, at $T=300 \mathrm{~K}$ and $\rho=0.998 \mathrm{~g} / \mathrm{cm}^{3}[8]$.

The time dependencies of the velocity autocorrelation function $\left\langle v_{\alpha}(t) v_{\alpha}(0)\right\rangle m / k_{B} T$ and of the diffusion coefficient

\footnotetext{
*Permanent address: Karpov Institute of Physical Chemistry, Ul. Vorontsovo Pole 10, 103064, Moscow K-64, Russia. Email address: berezh@speck.niddk.nih.gov

${ }^{\dagger}$ Email address: g.sutmann@fz-juelich.de
}

$D(t)=0.5 d\left\langle x^{2}(t)\right\rangle / d t=\int_{0}^{t} d t^{\prime}\left\langle v_{\alpha}\left(t^{\prime}\right) v_{\alpha}(0)\right\rangle$, shown in Fig. 1 , suggest that diffusion is a proper language for times larger than 2 ps. Similar estimates can be found in the literature, see, for example, p. 192 in Ref. [1]. It will be shown, however, that these estimates are too optimistic and strong deviations from the behavior predicted by the diffusion theory occur at much longer times.

To decide whether the motion can be described in terms of diffusion or not, one can compare the exact propagator found by simulations with the propagator predicted by the diffusion theory (using the diffusion constant found by simulations). Alternatively one can compare the moments of the displacement. We choose the second way and consider the
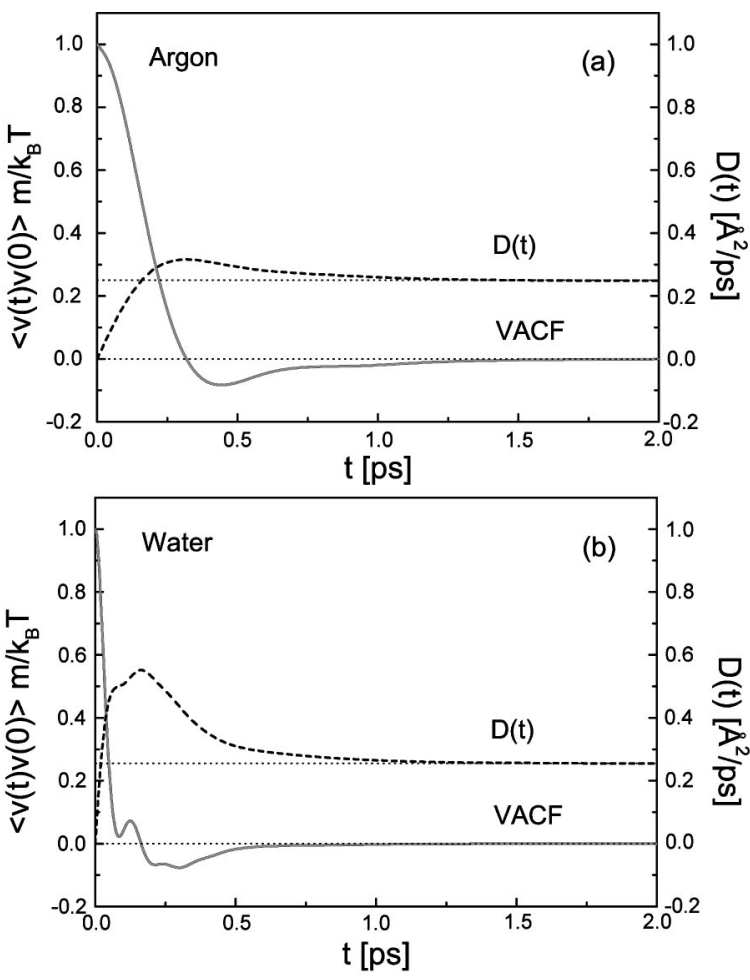

FIG. 1. The normalized velocity autocorrelation function (solid curve) and the time dependent diffusion coefficient $D(t)$ (dashed curve) for (a) argon and (b) water. 

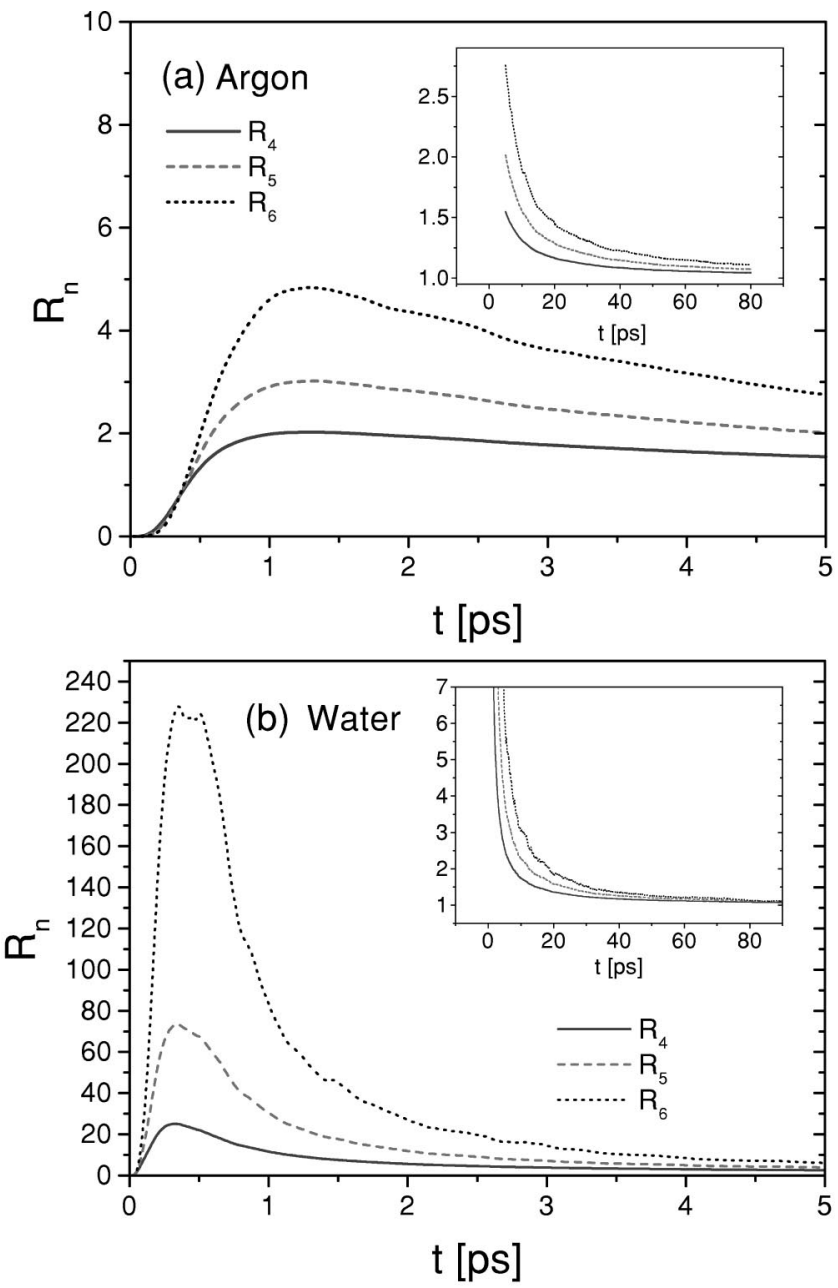

FIG. 2. The ratio $R_{n}(t)(n=4,5,6)$, characterizing the nondiffusion behavior for (a) argon and (b) water. The inset shows the $1 / t$ long time behavior.

ratio of the moment $\left\langle x^{2 n}(t)\right\rangle$ found by simulations to the same moment predicted by the diffusion theory, $\left\langle x^{2 n}(t)\right\rangle_{d}$ $=[(2 n-1) ! !](2 D t)^{n} . \quad$ This ratio, $\quad R_{n}(t)=\left\langle x^{2 n}(t)\right\rangle /$ $\left\langle x^{2 n}(t)\right\rangle_{d}$, tends to unity as $t \rightarrow \infty$ since the motion becomes diffusion at large times. At small times, when the motion is ballistic, $\left\langle x^{2 n}(t)\right\rangle \propto t^{2 n}$ and $R_{n}(t)$ vanishes as $t \rightarrow 0$. The ratio $R_{n}(t)$ is closely related to the non-Gaussian parameter $\alpha_{n}$ introduced by Rahman [9] and often used in studies of supercooled liquids and glasses.

Since a precise determination of higher moments of displacement is a delicate statistical problem, we have extended our MD calculations to rather long times. In the case of argon the length of the trajectory was $100 \mathrm{~ns}$, whereas in the case of water it was $30 \mathrm{~ns}$. In order to avoid statistical artifacts in the displacement calculation we choose a window method, where only after time intervals of $\Delta t=10 \mathrm{ps}$ a new displacement vector is filled for further analysis.

The dependencies $R_{n}(t)$ are nonmonotonic as shown in Fig. 2. The ratio grows rapidly at small $t$, reaches its maximal value, and then decreases slowly to unity. The larger the $n$, the greater is the maximal value of $R_{n}(t)$. The deviations from what diffusion theory predicts are much stronger for
TABLE I. Ratio $R_{n}(t)$ between the moments of the displacement found from simulation data and predicted by the diffusion theory at time $t=2 \mathrm{ps}$.

\begin{tabular}{lccccc}
\hline \hline & $R_{2}$ & $R_{3}$ & $R_{4}$ & $R_{5}$ & $R_{6}$ \\
\hline $\mathrm{H}_{2} \mathrm{O}$ & 1.75 & 3.0 & 5.6 & 11.9 & 27.3 \\
$\mathrm{Ar}$ & 1.15 & 1.4 & 1.9 & 2.8 & 4.4 \\
\hline \hline
\end{tabular}

water than for argon. The maximum value of $R_{6}(t)$ is approximately 220 in water, while in argon it is $\approx 5$. Note that in water $R_{6}(t)$ reaches its maximum at times that are very similar to the characteristic lifetime of the hydrogen bond [10]. Table I gives the ratio at $t=2 \mathrm{ps}$ when the diffusion coefficient has reached the plateau value (Fig. 1).

As $t \rightarrow \infty, R_{n}(t)$ slowly approaches unity. We found that at times between $10 \mathrm{ps}$ and $80 \mathrm{ps}$ all $R_{n}(t)$ are well represented by the dependence

$$
R_{n}(t)=\frac{\left\langle x^{2 n}(t)\right\rangle}{[(2 n-1) ! !](2 D t)^{n}}=1+\frac{b_{n}}{t}
$$

with coefficients $b_{n}$ given in Table II. Figure 3 illustrates the quality of this approximation. At $t=10 \mathrm{ps}$, deviations from the results predicted by the diffusion theory approximately are $18 \%$ and $5 \%$ in $R_{2}, 40 \%$ and $16 \%$ in $R_{3}, 72 \%$ and $33 \%$ in $R_{4}, 118 \%$ and $56 \%$ in $R_{5}$, and $183 \%$ and $87 \%$ in $R_{6}$ for water and argon, respectively. Thus the deviations are well pronounced at $t=10 \mathrm{ps}$. Note that the long time tail discussed in the present paper should not be confused with the long time tail in the decay of the velocity autocorrelation function discovered by Alder and Wainwright [11].

In order to rationalize the $1 / t$ behavior of $R_{n}(t)$ we use a continuous time random walk (RW) model for molecular motion. We assume that the random walk is Markovian and the probability density for the waiting time between successive steps is exponential, i.e., $\phi(t)=k \exp (-k t)$, where $k^{-1}$ is the average waiting time. The second characteristic of the random walk is the probability density for the step displacement (sd), $p(x)$, which is assumed to be a symmetric function of $x$, i.e., $p(x)=p(-x)$. Consequently all odd moments of the displacement are zero while even moments are finite,

$$
\left\langle x^{2 n}(t)\right\rangle_{s d}=\int_{-\infty}^{\infty} x^{2 n} p(x) d x .
$$

For this random walk the diffusion constant is found to be $D=k\left\langle x^{2}\right\rangle_{s d} / 2$. For large $t, k t \gg 1$, we find

TABLE II. Coefficients $b_{n}$ from Eq. (1). Errors (shown in parentheses) were estimated by fitting the $R_{n}(t)$ over different time intervals.

\begin{tabular}{lccccc}
\hline \hline & $b_{2}$ & $b_{3}$ & $b_{4}$ & $b_{5}$ & $b_{6}$ \\
\hline $\mathrm{H}_{2} \mathrm{O}$ & $1.8(0.1)$ & $4.0(0.1)$ & $7.2(0.2)$ & $11.85(1.0)$ & $18.3(2.8)$ \\
$\mathrm{Ar}$ & $0.45(0.02)$ & $1.54(0.08)$ & $3.2(0.1)$ & $5.7(0.1)$ & $8.9(0.1)$ \\
\hline \hline
\end{tabular}



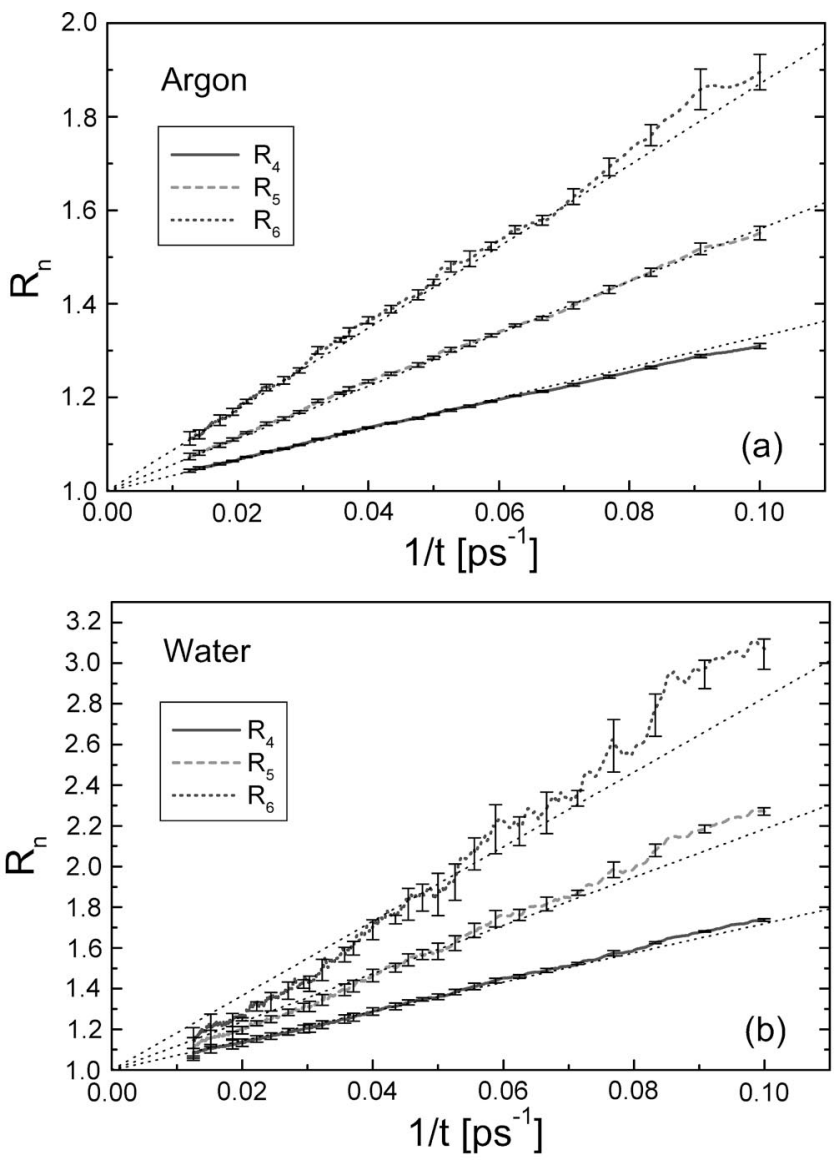

FIG. 3. The ratio $R_{n}(t)(n=4,5,6)$ vs inverse time, demonstrating the $1 / t$ behavior for (a) argon and (b) water. Dotted lines represent Eq. (1) with coefficients $b_{n}$ taken from Table II. Error bars are shown exemplary for selected points.

$$
\left\langle x^{2 n}(t)\right\rangle_{R W} \simeq[(2 n-1) ! !](2 D t)^{n}\left[1+\frac{n(n-1)\left\langle x^{4}\right\rangle_{s d}}{6\left\langle x^{2}\right\rangle_{s d}^{2} k t}\right] .
$$

This leads to $R_{n}(t)$ of the form given in Eq. (1) with

$$
\left.b_{n}\right|_{R W}=\frac{n(n-1)\left\langle x^{4}\right\rangle_{s d}}{6 k\left\langle x^{2}\right\rangle_{s d}^{2}} .
$$

According to Eq. (4) the ratio between coefficients is simply given by

$$
\frac{b_{n+1}}{b_{n}}=\frac{(n+1)}{(n-1)}
$$

independent of the parameters of the random walk. To test this prediction we calculated the ratio $b_{n+1} / b_{n}$ using $b_{n}$ from Table II. The results, given in Table III, show that the random walk model not only correctly predicts the long time behavior of $R_{n}(t)$ in Eq. (1), but it also reasonably well predicts the ratio of the coefficients $b_{n+1} / b_{n}$.

Thus, the simulations suggest that between the exact many-body description of motion of molecules in liquids and
TABLE III. Ratio of coefficients $b_{n}$ [c.f. Eq. (1)] predicted by the random walk model and found from simulations for water and argon. Error estimates are given in parentheses.

\begin{tabular}{lcccc}
\hline \hline & $b_{3} / b_{2}$ & $b_{4} / b_{3}$ & $b_{5} / b_{4}$ & $b_{6} / b_{5}$ \\
\hline $\mathrm{RW}$ & 3 & 2 & 1.66 & 1.5 \\
$\mathrm{H}_{2} \mathrm{O}$ & $2.19(0.1)$ & $1.8(0.1)$ & $1.64(0.15)$ & $1.56(0.27)$ \\
$\mathrm{Ar}$ & $3.4(0.2)$ & $2.1(0.14)$ & $1.74(0.08)$ & $1.58(0.04)$ \\
\hline \hline
\end{tabular}

the hydrodynamic one based on the diffusion equation, there is an intermediate region where the motion is described in terms of a random walk.

To get an idea of the parameters of the random walk we assume that all steps have the same length $l$ and all directions for the step are equally probable, i.e., we consider Pearson's random walk [12]. In this case $\left\langle x^{4}\right\rangle_{s d} /\left\langle x^{2}\right\rangle_{s d}^{2}=1.8$ and the expression in Eq. (4) takes the form $b_{n}=0.3 n(n-1) / k$. This relation can be used to estimate the average time $k^{-1}$ $=10 b_{n} /[3 n(n-1)]$. Using $b_{n}$ given in Table II we obtain $k^{-1} \simeq 2$ ps for water and $k^{-1} \simeq 1$ ps for liquid argon. We use these $k^{-1}$ and the diffusion constants found in our simulations, $D_{\mathrm{H}_{2} \mathrm{O}}=0.256 \AA^{2} / \mathrm{ps}$ and $D_{\mathrm{Ar}}=0.253 \AA^{2} / \mathrm{ps}$, to estimate the step length by the formula $l=\sqrt{6 D k^{-1}}$ (here we have used the fact that for Pearson's random walk $\left\langle x^{2}\right\rangle_{s d}$ $=l^{2} / 3$ ). This leads to $l_{\mathrm{H}_{2} \mathrm{O}} \simeq 1.7 \AA$ and $l_{\mathrm{Ar}} \simeq 1.3 \AA$.

The length and times associated with the random walk are too small to describe jumps of an individual molecule. We believe that the random walk is due to jumps of the liquid's configuration from one local minimum of the multidimensional potential energy surface (cell) to another as was suggested by Zwanzig [5]. From an individual particle point of view, jumps among cells lead to rearrangements of its equilibrium position. After such a jump the particle starts relaxation to a new equilibrium. Effectively this can be described in terms of random walk with a waiting time that corresponds to the time required for the liquid configuration to change. It is not surprising that such random walk has a short waiting time and small step length. We believe that this picture is quite generic and random walk due to jumps among the energy minima underlies self-diffusion not only in water and liquid argon.

Finally we discuss the relation between our work and Cao's analysis of diffusion [13] with the diffusion coefficient that randomly jumps between two values. In this problem an exact propagator reduces to an ordinary diffusive propagator with an effective diffusion constant as $t \rightarrow \infty$. However, when $t$ is not large enough there is a difference between the exact and the effective diffusive propagators. It is interesting that both exact and effective propagators predict the same mean square displacement at all times. But there is a difference in higher moments that vanishes as $t \rightarrow \infty$. Cao analyzed the difference in the fourth moments. He showed that the difference approaches zero as $1 / t$ if the probability density for the waiting time for jumps between different values of the diffusion coefficient is exponential. Thus, Cao found the same $1 / t$ relaxation to diffusive behavior in a related, but quite different problem. 
In summary, in order to establish the range of applicability of diffusion for describing motion of molecules in liquids, the higher moments $(4-12)$ of the displacement were found by MD computer simulations and compared with the moments predicted by the diffusion theory. The comparison showed that the difference slowly decreases with time according to a $1 / t$ law. This $1 / t$ behavior can be explained if one assumes the existence of a random walk that after sufficiently many steps leads to diffusion. In this paper we analyzed water and argon at some specific conditions. It will be interesting to study whether the behavior we found is universal for a broader class of liquids and a wider range of thermodynamic state points. From our present investigation we conclude that one has to be careful when using the language of diffusion to describe motion of molecules on times of the order of tens picoseconds and lengths of the order of $5 \AA$. In our opinion it is better to describe molecular/atomic motion in this time and length regime in terms of random walk.

A.M.B. is thankful to A. Szabo and R. Zwanzig for numerous and very useful discussions.
[1] U. Balucani and M. Zoppi, Dynamics of the Liquid State (Clarendon Press, Oxford, 1994).

[2] P.A. Egelstaff, An Introduction to the Liquid State (Clarendon Press, Oxford, 1992).

[3] J.P. Hansen and I.R. McDonald, Theory of Simple Liquids (Academic Press, London, 1986).

[4] J.P. Boon and S. Yip, Molecular Hydrodynamics (Dover, New York, 1980).

[5] R. Zwanzig, J. Chem. Phys. 79, 4507 (1983).

[6] E. Rabani, J.D. Gezelter, and B.J. Berne, J. Chem. Phys. 107, 4618 (1997); J.D. Gezelter, E. Rabani, and B.J. Berne, ibid. 110, 3444 (1999).

[7] H.J.C. Berendsen, J.R. Grigera, and T.P. Straatsma, J. Phys. Chem. 91, 6269 (1987)
[8] All simulations were performed in the microcanonical ensemble under periodic boundary conditions. In the case of water we used a reaction field method to treat the long range Coulomb interactions. The time step of integration was $\delta t$ $=20$ fs and $\delta t=1$ fs for argon and water, respectively.

[9] A. Rahman, Phys. Rev. 136, A405 (1964).

[10] A. Luzar and D. Chandler, Nature (London) 379, 55 (1996); G. Sutmann and R. Vallauri, J. Mol. Liq. 98-99, 215 (2002).

[11] B.J. Alder and T.E. Wainwright, J. Phys. Soc. Jpn. 26, 267 (1969); Phys. Rev. A 1, 18 (1970).

[12] G.H. Weiss, Aspects and Applications of the Random Walk (North-Holland, Amsterdam, 1994).

[13] J. Cao, Phys. Rev. E 63, 041101 (2001). 